\title{
Successful pulmonary embolectomy for massive embolism following pneumonectomy
}

\author{
Guven Olgac, MD., Ann June Patricia McKeown, FRCS, Michael Edward Cowen, FRCS \\ Department of Cardiothoracic Surgery, Castle Hill Hospital, Castle Road, Cottingham, HU16 5JQ, Hull, UK
}

\begin{abstract}
Introduction
Massive pulmonary embolism (MPE) can be a devastating complication following surgery and is associated with a high mortality due to severe haemodynamic instability. To date available treatment modalities are: 1 . medical management with heparin and/or thrombolytic agents, and 2. surgical management with pulmonary embolectomy via cardiopulmonary bypass (CPBP) followed by anticoagulation therapy with or without interruption of inferior vena cava. The success rate for both treatment modalities is similar and very much depends on the patient's clinical status that is judged by haemodynamic parameters ${ }^{1-3}$. Thrombolytic therapy is associated with a high incidence of bleeding following major surgery while the mortality from surgical management varies from $11 \%$ to $94 \%^{2,4}$.
\end{abstract}

\section{Case Report}

A 62 year-old female underwent an uneventful left pneumonectomy for T3N1M0 squamous cell carcinoma. She was extubated in theatre and was transferred to the Intensive Care Unit (ICU) where she made a good progress. On the first postoperative day, her drain was removed and she was transferred back to the ward with good haemodynamics. Later that day, she became very breathless, cold and clammy and her arterial oxygen saturation $\left(\mathrm{SaO}_{2}\right)$ suddenly dropped from $95 \%$ to $75 \%$. Clinical examination revealed signs of respiratory distress with a respiratory rate of 40 per minute and marked cyanosis. The patient was in a low cardiac

Address for correspondence:

Dr. Guven OLGAC

Zuhuratbaba Mah. Turanli Sok.

No: 4, D: 26, Bakirkoy 34147

Istanbul, Turkey

Phone : +90 (533) 4155434

Fax : $+90(212) 5472233$

E-mail : golgac@tnn.net

$@$ IJTCVS 097091342041204/092

Received -05/07/04; Review Completed - 16/08/04; Accepted - 26/08/04. output status with tachycardia, elevated jugular venous pressure and low systemic blood pressure (80/40 $\mathrm{mmHg}$ ). The trachea was slightly deviated towards the left side with normal vesicular breath sounds on the right. ECG proved sinus tachycardia $(110 / \mathrm{min})$ with no evidence of acute myocardial ischemia. Her CXR showed normal post-pneumonectomy changes with cut-off sign of right pulmonary artery at the level of right hilum, diminished vascular shadowing throughout the lung periphery and dilated right atrium (Fig. 1). Arterial blood gas analysis revealed $\mathrm{PaO}_{2}: 54 \mathrm{mmHg}, \mathrm{PaCO}_{2}: 48$ $\mathrm{mmHg}, \mathrm{pH}: 7.12, \mathrm{SaO}_{2}: 68 \%$. Supplemental oxygen via face mask failed to improve her gas exchange. She was therefore intubated on the ward and transferred back to the ICU. However, she remained in a low cardiac output status despite maximum inotropic and ventilatory support. A diagnosis of MPE was accomplished based on radiological findings and lack of clinical response to supportive medical treatment.

The patient was taken to the operating theatre for emergency pulmonary embolectomy two hours after initial event. She developed cardiac arrest during median sternotomy and CPBP was established with aorto-bicaval cannulation while continuing internal cardiac massage. Appearances were that of MPE with dilated right heart chambers and distended vena cavae. The main pulmonary artery was then opened and large amount of clot was removed from the right pulmonary artery. She came off bypass with minimal inotropic support and electively ventilated overnight in the ICU. The following day she was successfully weaned from the ventilator and inotrops and was finally extubated with good gas exchange. Anticoagulation was commenced with intravenous heparin and warfarin. In view of the rapid onset of the thromboembolism, and proven large amount of clot in the lower extremities by a Doppler ultrasound, an inferior caval filter was also inserted at the same day. CXR revealed restoration of right pulmonary artery shadowing at the right hilum and the lung periphery as well as relief of strain on the right atrial wall (Fig. 2). She made an uneventful recovery afterwards and was discharged home on $12^{\text {th }}$ postoperative day. 


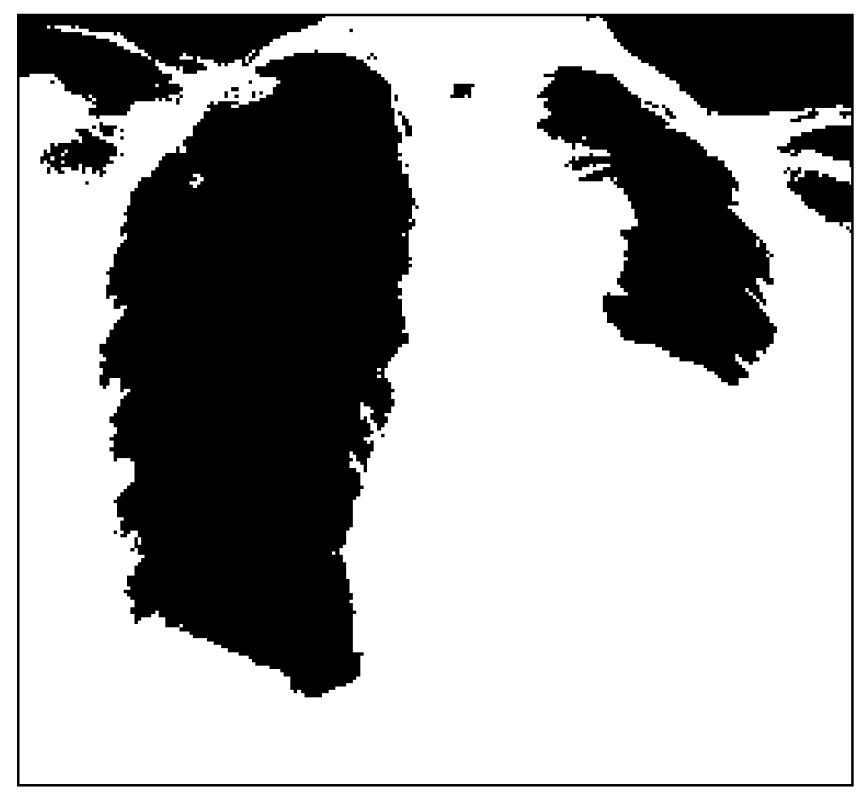

Fig. 1. CXR showing amputation of right pulmonary artery (white arrow) and diminished pulmonary vascular shadowing throughout the right lung periphery with normal post-pneumonectomy appearance on the left. Note distended right atrial wall (black arrows).

\section{Discussion}

Acute MPE is the commonest reason for death from pulmonary causes in hospital and a very important cause of death in surgical practice ${ }^{5}$. Thus, prompt diagnosis and surgical relief of the obstruction at the earliest possible opportunity is the most effective means for reducing the mortality from MPE. A careful clinical examination together with simple and quick diagnostic tools like CXR, ECG, arterial blood gas analysis and measurement of the arterial and central venous pressures are usually sufficient to exclude other possible causes of low cardiac output and poor tissue oxygenation. Because of unnecessary delay and subsequent death, more sensitive but time consuming investigations such as pulmonary angiography and $\mathrm{V} / \mathrm{Q}$ scan of the lungs are not always mandatory for diagnosis ${ }^{5-7}$.

Among all treatment modalities, anticoagulation with heparin has the lowest success rate and should therefore be reserved for those who have minimal or no circulatory impairment ${ }^{2}$. Thrombolytic therapy and pulmonary embolectomy have roughly equal but variable outcomes that depend on the patient's haemodynamic status. Although associated with a high incidence of bleeding in the early postoperative period, thrombolysis is highly effective in the management of "subacute" MPE and therefore is considered a relative

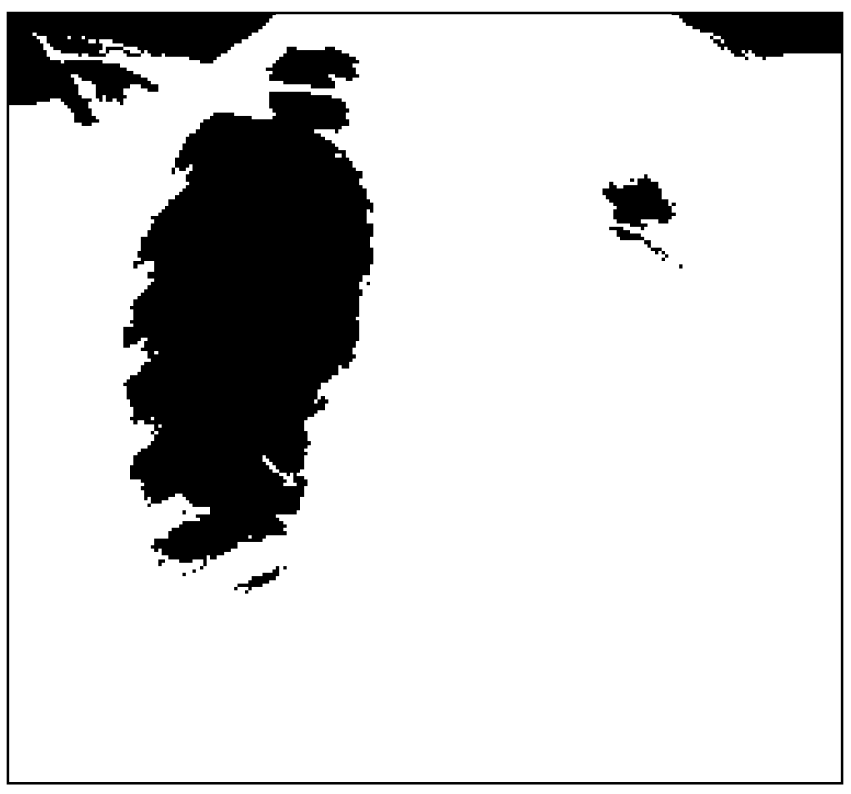

Fig. 2. CXR following pulmonary embolectomy showing restoration of right pulmonary artery shadowing at the right hilum (white arrows) and the lung periphery with normal right atrial shadow.

rather than an absolute contraindication after a major surgery ${ }^{8}$. Nevertheless, it is the only alternative therapeutic modality when there is no available cardiothoracic unit nearby. Pulmonary embolectomy via CPBP however, immediately restores fatal haemodynamic instability and gas exchange and also reduces the need for inotropic and ventilatory support secondary to dramatic relief of the strain on the right heart as seen in our patient. Interruption of the inferior vena cava with a transvenous filter and anticoagulation therapy prevent further thromboembolic events.

The time elapsed between onset of the symptoms and therapeutic intervention is also very important as no compensation mechanism exists after MPE following a pneumonectomy. Therefore, a high level of suspicion should be maintained for any patient that suffers from severe haemodynamic compromise postoperatively. Prompt embolectomy without delay may be life-saving in such cases even following pneumonectomy, since there does not seem to be an effective and safe alternative treatment.

\section{References}

1. Clarke DB, Abrams LD. Pulmonary embolectomy: a 25 year experience. J Thorac Cardiovasc Surg 1986; 92 (3) : 442-45.

2. Miller GAH, Hall RJC, Paneth M. Pulmonary embolectomy, heparin, and streptokinase: Their place in the treatment of acute massive pulmonary embolism. Am Heart J 1977; 93 (5): 568-74. 
3. Stulz P. Schlapfer R, Feer R, Habicht J, Gradel E. Decision making in the surgical treatment of massive pulmonary embolism. Eur $J$ Cardiothorac Surg 1994; 8(4): 188-93

4. Doerge H, Schoendube FA, Voss M, Seipelt R, Messmer BJ. Surgical therapy of fulminant pulmonary embolism: Early and late results. Thorac Cardiovasc Surg 1999; 47 (1): 9-13.

5. Kalweit G, Huwer H, Volkmer I, Petzold T, Gams E. Pulmonary embolism: A frequent cause of acute fatality after lung resection. Eur J Cardiothorac Surg 1996: 10 (4): 242-47.

6. Schmid C, Zietlow S, Wagner TO, Laas J, Borst HG. Fulminant pulmonary embolism: Symptoms, diagnostics, operative technique, and results. Ann Thorac Surg 1991; 52 (5): 1102-07.

7. Chen Q. Tang AT, Tsang GM. Acute pulmonary thromboem bolism complicating pneumonectomy: Successful operative management. Eur J Cardiothorac Surg 2001; 19 (2) : 223-25.

8. Girard P, Baldeyrou P, Le Guillou JL, Lamer C, Grunenwald D. Thrombolysis for life-threatening pulmonary embolism two days after lung resection. Am Rev Respir Dis 1993; 147 1595-97. 